\title{
Optimization of HO photonic crystal nanocavity using machine learning
}

\author{
Ryotaro Abe, Taichi Takeda, Ryo Shiratori, Shinichi Shirakawa, Shota \\ SAITO AND TOSHIHIKO BABA* \\ Department of Electrical and Computer Engineering, Yokohama National University, 79-5 Tokiwadai, Hodogayaku, Yokohama 240-8501, Japan \\ *Corresponding author: baba-toshihiko-zm@ynu.ac.jp
}

Received XX Month XXXX; revised XX Month, XXXX; accepted XX Month XXXX; posted XX Month XXXX (Doc. ID XXXXX); published XX Month XXXX

\begin{abstract}
Using machine learning, we optimized an ultrasmall photonic crystal nanocavity to attain a high $Q$. Training data was collected via finite-difference time-domain simulation for models with randomly shifted holes, and a fully connected neural network (NN) was trained, resulting in a coefficient of determination between predicted and calculated values of 0.977 . By repeating $N N$ training and optimization of the $Q$ value on the trained $N N$, the $Q$ was roughly improved by a factor of 10-20 for various situations. Assuming a 180-nm-thick semiconductor slab at a wavelength approximately 1550 nm, we obtained $Q=1,011,400$ in air; 283,200 in a solution, which was suitable for biosensing; 44,600 with a nanoslot for high sensitivity. Important hole positions were also identified using the linear Lasso regression algorithm. (c) 2019 Optical Society of America
\end{abstract}

http://dx.doi.org/10.1364/OL.99.099999

Photonic crystal (PC) nanocavities comprising a periodic array of holes with small irregularities in a high index slab have been studied for some decades because of their unique physics and ability to be employed as tools in other physics experiments and for demonstrating miniature photonic devices. In particular, an $\mathrm{HO}$ nanocavity formed only by shifting some holes and without missing any holes achieves an extremely small modal volume as a dielectric cavity indicating particularly dense light localization while maintaining a high $\mathrm{Q}$ factor [1]. Therefore, it has been utilized for laser operation [2, 3], biosensing [4], nonlinear switching [5], and cavity quantum electrodynamics $[6,7]$.

Enhancement of the Q factor in PC cavities has been extensively investigated as one of their crucial performance parameters [8]. In general, the $Q$ value depends on cavity size (or modal volume) and situations targeted in each study. For example, $Q$ depends on slab

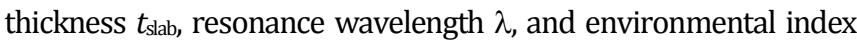
$n_{\text {env. }}$. The $Q$ factor is higher for a larger $t_{\text {slab }} / \lambda$ and a smaller $n_{\text {env. }}$. So far, $\mathrm{Q}$ of the $\mathrm{H} 0$ nanocavity has been calculated by using finite-difference time-domain (FDTD) method assuming $n_{\mathrm{env}}=1.0$ in air, and different values have been reported such as $Q=112,000$ with $t_{\text {slab }} / \lambda$ $=0.190$ [1], 760,000 with $t_{\text {slab }} / \lambda=0.139$ [7], and 130,000 with $t_{\text {slab }} / \lambda$ $=0.090$ [2]. In biosensing applications, a device is often operated in a solution (let us assume that the $n_{\text {env }}$ of the solution is the same as that of water, 1.321). Moreover, $t_{\text {slab }}$ is set to be small and a nanoslot is often introduced to improve the sensitivity. These conditions considerably reduce $Q$. If the target is a nanolaser biosensor similar to the one we have developed, $Q>10,000$ will be sufficient for laser operation with a standard active semiconductor. However, a much higher $\mathrm{Q}$ is preferable for robust operation in actual devices with some disordering induced during fabrication. The studies mentioned above optimized the position of some holes around the cavity to enhance $\mathrm{Q}$. Therefore, $\mathrm{Q}$ could be further enhanced by applying similar optimization to many holes. However, optimizing many parameters by repeating the time-consuming FDTD calculation is not straightforward.

(a)

(b)

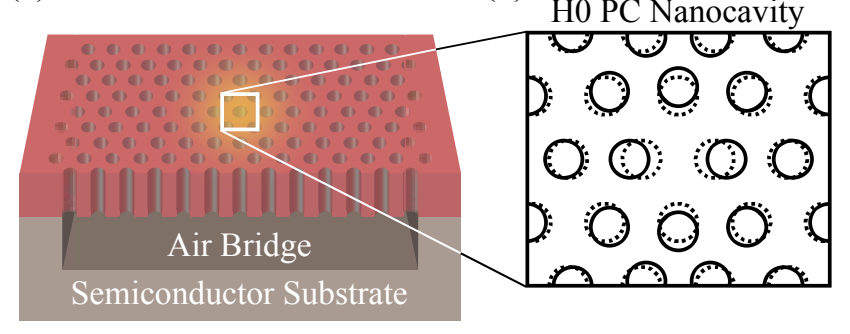

Fig. 1. Schematic of the H0 PC nanocavity. (a) Overview. (b) Magnified view of the hole arrangement around the nanocavity. The solid lines show the hole positions modified by machine learning, while the dotted lines show the original positions.

The inverse design concept is helpful in optimizing complicated photonics devices $[9,10]$, and some studies have recently employed machine learning [11-17]. Asano, et al., applied it to the optimization of a large PC cavity and calculated Q of the order $10^{9}$ [18]. In this study, we applied it to the $\mathrm{H} 0$ nanocavity focusing on its application 
to lasing and biosensing. Therefore, we assumed air or a solution as the environment and searched for a high $Q$ at a small $t_{\text {slab }} / \lambda$ with and without a nanoslot. In general, machine learning is categorized into linear and nonlinear models. Nonlinear models such as neural network (NN) and random forest regression produce more precise models than linear models, while linear models such as Ridge and Lasso regression clarify the contribution of explanatory variables to the target variable. In this study, we tested all these models and finally used a fully connected NN model to find an optimized structure. We also analyzed the correlation between Q and hole positions using the Lasso regression model; the Lasso model expresses the $Q$ value as a linear combination of the parameters whose coefficients represent to the partial regression.
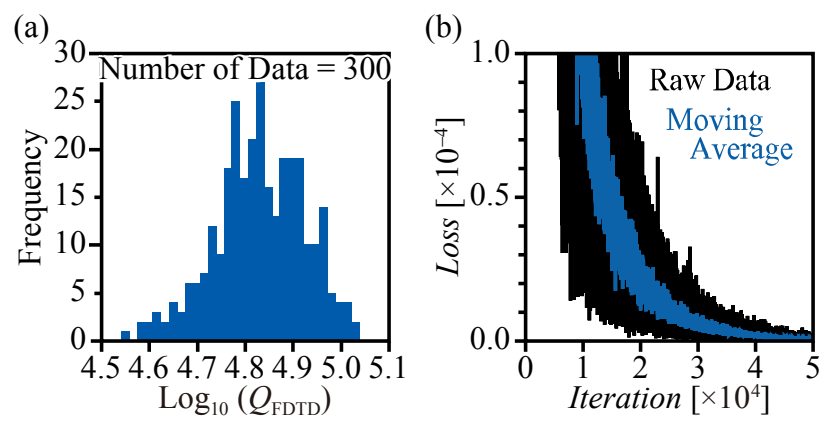

(c)

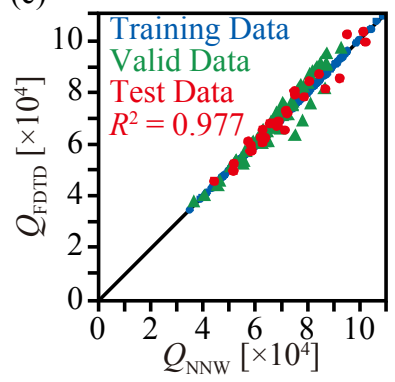

Fig. 2. The training process of NN using collected data of the $Q$ factor. (a) Distribution of collected data. (b) Transition of error between calculated and predicted values. (c) Relation between Q calculated by FDTD and predicted by NNW.

The basic structure of the H0 nanocavity comprised a triangular lattice PC slab with an index $n_{\text {slab }}=3.4$, normalized thickness $t_{\text {slab }} / \lambda$ $=0.106-0.115$, lattice constant $a=500 \mathrm{~nm}$, a hole diameter $2 r=260$ $\mathrm{nm}$, and the shift of the two holes from the center to the outside by $80 \mathrm{~nm}$, which referred to the GaInAsP PC nanolaser operating at $\lambda \approx$ $1550 \mathrm{~nm}$, was included [2]. First, 38-62 holes around the cavity center, which overlapped the major modal electric field, were randomly shifted in the $x$ and $y$ directions within a maximum range of $\pm 5 \mathrm{~nm}$, and the $\mathrm{Q}$ values were collected as training data using Lumerical FDTD Solutions. Figure 2(a) shows the logarithmic distribution of 300 data collected, which exhibits a Gaussian-like distribution. The data is split into training data for model training, validation data for hyperparameter tuning, and test data for evaluating the performance of the NN model. The NN employed in Fig. 2 consists of an input layer corresponding to the shift amounts of all the holes, two hidden layers each having 300 neurons, and an output layer to give logarithmic Q. We used a stochastic gradient descent method with a learning rate of 0.01 to train the NN model.
Figure 2(b) shows the transition of the squared error for the training data. The error was converged when the number of iterations exceeds 30,000. Figure 2(c) shows the relation between the values calculated by the FDTD and those predicted by the NN. The coefficient of determination $R^{2}$ for the test data was as high as 0.977 indicating that the NN model well represented the behavior of $\mathrm{Q}$ in the $\mathrm{HO}$ nanocavity. We also applied the Lasso regression model to the similar dataset and confirmed a similarly high coefficient of determination of 0.960 when the coefficient of the L1 norm was $10^{-5}$.

Based on this first trained NN or Lasso model, we searched for the promising structure being expected to show a higher $Q$ value. In this process, we first changed only one parameter of the structure randomly and obtained a value of the parameter which maximized the $Q$ value. Next, we fixed this parameter, and then changed another parameter and did the same. We repeated this procedure for all parameters and finally obtained a maximum $Q$. As this $Q$ value depends on the order of changing parameters, we tried the same procedure by randomly changing the order and obtained some high $\mathrm{Q}$ candidates. Finally, we verified their values by the FDTD and determined the best structure and highest Q. As the next step, we collected additional data for similar random shifts, regarding the soobtained best structure as the next basic structure and repeated the same learning and searching. We repeated the steps several times with the data to 4,300 in total.

Figure 3 shows example structures obtained by these models. Black and red lines show the hole positions of the basic and optimized structures, respectively. The shift directions of each hole are not necessarily the same between the two models', but the shift amounts are small in both cases. The $Q$ value was improved by shifting some holes around the cavity and slightly shifting the holes apart from the cavity in the diagonal directions along the mode penetration. The $Q$ value was improved as the number of shifted holes was increased. Figure 3(c) shows the cavity mode profile of the optimized structure, which was almost unchanged from the basic structure. Figure 3(d) visualizes the normalized partial regression coefficients obtained by Lasso (in the color map). We defined $\sqrt{(}\left(R_{\mathrm{x}}{ }^{2}+R_{\mathrm{y}}{ }^{2}\right)$ as the correlation value of each hole, where $R_{\mathrm{x}}$ and $R_{\mathrm{y}}$ are the partial regression coefficients for $x$ and $y$, respectively. In Fig. 3(d), it is plotted after normalized by their maximum. In general, the holes with larger correlation values are considered as important holes for the $\mathrm{Q}$ value. We confirmed a particularly high regression coefficient at six holes around the cavity. The coefficient in the diagonal directions where the mode deeply penetrated was also high. It is seen in the comparison between Figs. 3(b) and (d) that the shifts of the six holes around the cavity center are very small, while their correlation values are large. This is because their positions of the basic structure were already close to their optima.

The highest $Q$ values obtained by using NN are summarized in Table 1 when different $t_{\text {slab }}$ and $n_{\text {env }}$ are assumed. After the optimization, Q increased from its initial value by a factor of 11.718.7. As a result of the optimization, $Q$ reached a value of over one million in air, and even though the value of $Q$ reduced in a solution, it was still as high as 283,200 . The values obtained by the Lasso model were roughly $20 \%$ smaller than these values, but still indicated an improvement. 
(a)

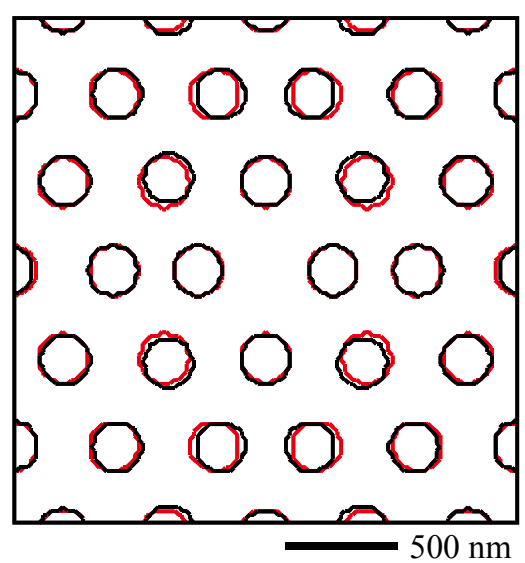

(b)

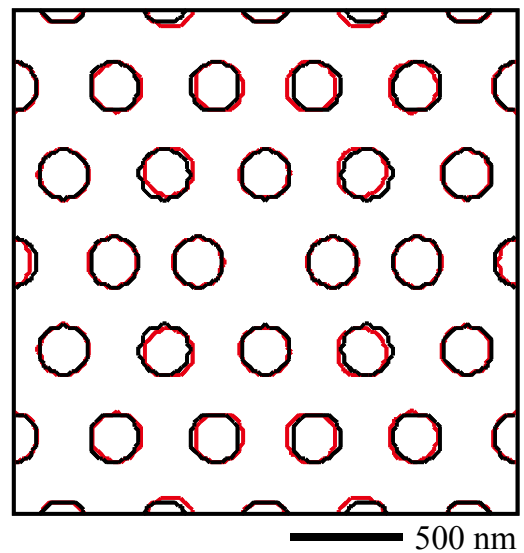

(c)

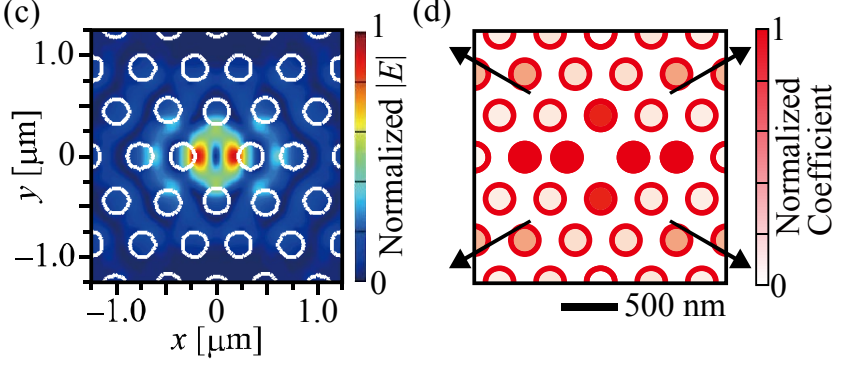

Fig. 3. The H0 nanocavity without nanoslot in a solution optimized by NN. (a), (b) The hole positions before (black) and after (red) optimization, respectively, which were obtained by using NN and Lasso regression models. (c) Mode profile after the NN optimization. (d) Visualized coefficient strength between $Q$ and shift amount of each hole, which was estimated by the Lasso model. Four arrows indicate the directions of mode penetration.

Table 1. The highest $\mathrm{Q}$ values of the $\mathrm{H} 0$ nanocavity without a nanoslot, which were calculated based on the NN model under different conditions.

\begin{tabular}{cccc}
\hline$t_{\text {slab }} / \lambda$ & $n_{\text {env }}$ & Initial Q & Optimized Q \\
\hline 0.106 & 1.0 & 46,400 & 779,000 \\
0.115 & 1.0 & 54,200 & $1,011,400$ \\
0.101 & 1.321 & 16,600 & 194,200 \\
0.110 & 1.321 & 19,600 & 283,200 \\
\hline
\end{tabular}

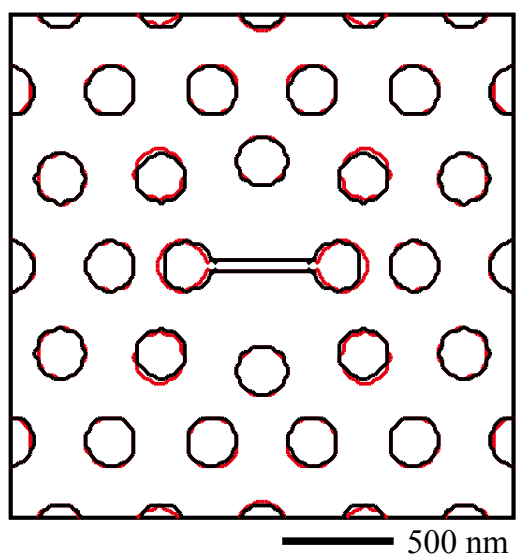

(b)

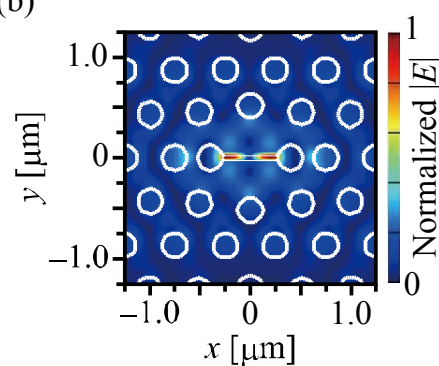

Fig. 4. H0 nanocavity with nanoslot in a solution, optimized by NN. (a) Hole positions before (black) and after (red) optimization. (b) Mode profile after the optimization.

Finally, we optimized the H0 nanocavity with a nanoslot, which was effective for athermal operation and high sensitivity $[3,4,19]$. In general, introducing a nanoslot considerably reduces the $Q$ value. Assuming $t_{\text {slab }}=180 \mathrm{~nm}, n_{\text {env }}=1.321$, and a slot width of $50 \mathrm{~nm}$, which is experimentally easy to fabricate, the calculated $Q$ value is only 4,600. We applied the same strategy as mentioned before and collected more than 1,000 data. Figure 4 shows structures before and after the optimization as well as the mode profile. The $Q$ value increased to 44,600 (9.7 times). This value is considerably lower than those in Table 1 without nanoslot, but sufficient to obtain the robust operation as a nanolaser.

In summary, we optimized the structure of an ultrasmall H0 PC nanocavity using machine learning and improved the calculated $Q$ factor in air and in a solution with and without nanoslot, and for a relatively thin slab. A Q value of over one million in air is the highest value ever reported for the $\mathrm{H} 0$ nanocavity. The $\mathrm{Q}$ value increased to roughly 10-20 times after the optimization for the different situations tested. We can now expect to obtain a more robust nanolaser suitable for biosensing. Compared with other inverse optimizations of photonic devices, machine learning works particularly well for the optimization of PC devices. Even though a PC has many holes as optimized parameters, defining them is considerably simpler than other photonic devices with smooth and complicated shapes.

Funding. Japan Society for the Promotion of Science (\#16H06334)

Disclosures. The authors declare that there are no conflicts of interest related to this article. 


\section{References}

1. Z. Zhang, and M. Qiu, Opt. Express 12, 3988 (2004).

2. K. Nozaki, S. Kita, and T. Baba, Opt. Express 15, 7506 (2007).

3S. Kita, K. Nozaki, S. Hachuda, H. Watanabe, Y. Saito, S. Otsuka, T. Nakada, Y. Arita, and T. Baba, IEEE J. Sel. Top. Quantum Electron. 17, 1632 (2011).

4. T. Baba, Photonics 6, 65 (2019).

5. K. Nozaki, T. Tanabe, A. Shinya, S. Matsuo, T. Sato, H. Taniyama, and M. Notomi, Nat. Photonics 4, 477 (2010).

6. M. Nomura, K. Tanabe, S. Iwamoto, and Y. Arakawa, Phys. Stat. Sol. C 8, 340 (2011).

7. Y. Ota, D. Takamiya, R. Ohta, H. Takagi, N. Kumagai, S. Iwamoto, and Y. Arakawa, Appl. Phys. Lett. 112, 093101, (2018).

8. T. Asano, Y. Ohchi, Y. Takahashi, K. Kishimoto, and S. Noda, Opt. Express 25, 1769 (2017).

9. T. Hashimoto, T. Saida, I. Ogawa, M. Kohtoku, T. Shibata, and H. Takahashi, Opt. Lett. 30, 2620 (2005).

10. A. Y. Piggott, J. Lu, K. G. Lagoudakis, J. Petykiewicz, T. M. Babinec, and J. Vučković, Nat. Photonics 9, 374 (2015).

11. Y. Shen, N. C. Harris, S. Skirlo, M. Prabhu, T. Baehr-Jones, M. Hochberg, X. Sun, S. Zhao, H. Larochelle, D. Englund, and M. Soljačić, Nat. Photonics 11, 441 (2017).

12. X. Lin, Y. Rivenson, N. T. Yardimci, M. Veli, Y. Luo, M. Jarrahi, and A. Ozcan, Science 361, 1004 (2018).

13. J. Bueno, S. Maktoobi, L. Froehly, I. Fischer, M. Jacquot, L. Larger, and D. Brunner, Optica 5, 756 (2018).

14. T. F. de Lima, H. T. Peng, A. N. Tait, M. A. Nahmias, H. B. Miller, B. J. Shastri, and P. R. Prucnal, J. Lightwave Technol., 37, 1515 (2019).

15. I. Malkiel, M. Mrejen, A. Nagler, U. Arieli, L. Wolf, and H. Suchowski, Light Sci. Appl. 7, 60 (2018).

16. J. Zhoou, B. Huang, Z. Yan, and J. G. Bünzli, Light Sci. Appl. 8, 84 (2019).

17. Y. Kiarashinejad, S. Abdollahramezani, M. Zandehshahvar, O. Hemmatyar, and A. Adibi, Adv. Theory Sim. 2, 1900088 (2019).

18. T. Asano, and S. Noda, Opt. Express 26, 32704 (2018).

19. S. Kita, S. Hachuda, K. Nozaki, and T. Baba, Appl. Phys. Lett. 97, 161108 (2010). 


\section{Full References}

1. Z. Zhang, and M. Qiu, "Small-volume waveguide-section high Q microcavities in 2D photonic crystal slabs", Opt. Express, vol. 12, no. 17, pp. 3988-3995, 2004.

2. K. Nozaki, S. Kita, and T. Baba, "Room temperature continuous wave operation and controlled spontaneous emission in ultrasmall photonic crystal nanolaser", Opt. Express, vol. 15, no. 12, pp. 7506-7514, 2007.

3. S. Kita, K. Nozaki, S. Hachuda, H. Watanabe, Y. Saito, S. Otsuka, T. Nakada, Y. Arita, and T. Baba, "Photonic crystal point-shift nanolaser with and without nanoslots --- design, fabrication, lasing and sensing characteristics", IEEE J. Sel. Top. Quantum Electron., vol. 17, no. 6, pp. 1632-1647, 2011.

4. T. Baba, "Photonic and iontronic sensing in GalnAsP semiconductor photonic crystal nanolasers", Photonics, vol. 6, no. 65, pp. 1-17, 2019.

5. K. Nozaki, T. Tanabe, A. Shinya, S. Matsuo, T. Sato, H. Taniyama, and M. Notomi, "Sub-femtojoule all-optical switching using a photonic-crystal nanocavity", Nature Photonics, vol. 4, pp. 477-483, 2010.

6. M. Nomura, K. Tanabe, S. Iwamoto, and Y. Arakawa, "Design of a high-Q HO photonic crystal nanocavity for cavity QED", Phys. Stat. Sol. C, vol. 8, no. 2, pp. 340-342, 2011.

7. Y. Ota, D. Takamiya, R. Ohta, H. Takagi, N. Kumagai, S. Iwamoto, and Y. Arakawa, "Large vacuum Rabi splitting between a single quantum dot and an H0 photonic crystal nanocavity", Appl. Phys. Lett. Vol. 112, no. 11, pp. 093101, 2018.

8. T. Asano, Y. Ohchi, Y. Takahashi, K. Kishimoto, and S. Noda, "Photonic crystal nanocavity with a Q factor exceeding eleven million", Opt. Express, vol. 25, no. 3, pp. 1769-1777, 2017.

9. T. Hashimoto, T. Saida, I. Ogawa, M. Kohtoku, T. Shibata, and H. Takahashi, "Optical circuit design based on a wavefront-matching method", Opt. Lett., vol. 30, no. 19, pp. 2620-2622, 2005.

10. A. Y. Piggott, J. Lu, K. G. Lagoudakis, J. Petykiewicz, T. M. Babinec, and J. Vučković, "Inverse design and demonstration of a compact and broadband on-chip wavelength demultiplexer", Nature Photonics, vol. 9, pp. 374-377, 2015.

11. Y. Shen, N. C. Harris, S. Skirlo, M. Prabhu, T. Baehr-Jones, M. Hochberg, X. Sun, S. Zhao, H. Larochelle, D. Englund, and M. Soljačić, “Deep learning with coherent nanophotonic circuits", Nature Photon., vol. 11, pp. 441446, 2017.

12. X. Lin, Y. Rivenson, N. T. Yardimci, M. Veli, Y. Luo, M. Jarrahi, and A. Ozcan, "All-optical machine learning using diffractive deep neural networks", Science, vol. 361, pp. 1004-1008, 2018.

13. J. Bueno, S. Maktoobi, L. Froehly, I. Fischer, M. Jacquot, L. Larger, and D. Brunner, "Reinforcement learning in a large-scale photonic recurrent neural network", Optica, vol. 5,no. 6, pp. 756-760, 2018.

14. T. F. de Lima, H. T. Peng, A. N. Tait, M. A. Nahmias, H. B. Miller, B. J. Shastri, and P. R. Prucnal, "Machine Learning With Neuromorphic Photonics", J. Lightwave Technol., vol. 37, no. 5, pp. 1515-1534, 2019.

15. I. Malkiel, M. Mrejen, A. Nagler, U. Arieli, L. Wolf, and H. Suchowski, "Plasmonic nanostructure design and characterization via Deep learning", Light Sci. Appl., vol. 7, no. 60, 2018.

16. J. Zhoou, B. Huang, Z. Yan, and J. G. Bünzli, "Emerging role of machine learning I light-matter interaction”, Light Sci. Appl., vol. 8, no. 84, 2019.

17. Y. Kiarashinejad, S. Abdollahramezani, M. Zandehshahvar, O. Hemmatyar, and A. Adibi, "Deep learning reveals underlying physics of light-matter interactions in nanophotonic devices," Adv. Theory Simulations vol. 2, pp. 1900088, 2019.

18. T. Asano, and S. Noda, "Optimization of photonic crystal nanocavities based on deep learning", Opt. Express, vol. 26, no. 25, pp. 32704-32716, 2018.

19. S. Kita, S. Hachuda, K. Nozaki, and T. Baba, "Nanoslot laser", Appl. Phys. Lett., vol. 97, no. 16, pp. 161108, 2010. 